CHRONIC OBSTRUCTIVE PULMONARY DISEASE

\title{
Overnight prescription of oxygen in long term oxygen therapy: time to reconsider the guidelines?
}

M Nisbet, T Eaton, C Lewis, W Fergusson, J Kolbe

Background: Guidelines for long term oxygen therapy (LTOT) recommend increasing oxygen flow by $1 \mathrm{l} / \mathrm{min}$ overnight. A study was undertaken in patients with COPD on LTOT to determine the prevalence of overnight desaturation if the usual oxygen flow rate is not increased at night, whether resting oxygen saturation predicts overnight desaturation, and whether overnight desaturation correlates with health related quality of life (HRQL) and sleep quality.

Methods: A cross sectional prospective study was performed on consecutive patients with COPD on LTOT attending our regional outpatient oxygen service. All patients fulfilled standard criteria for LTOT, had been established on LTOT at a flow to achieve resting oxygen saturations $>90 \%$, but had not been instructed to increase oxygen flow overnight. Overnight desaturation was defined as $<90 \%$ for $\geqslant 30 \%$ of the night on either of two consecutive nights. HRQL was evaluated with the SF-36 Health Survey Questionnaire, Chronic Respiratory Questionnaire, and the Pittsburgh Sleep Quality Index.

See end of article for authors' affiliations

Correspondence to: Dr T Eaton, Green Lane Respiratory Services, Auckland City Hospital, Auckland, New Zealand; teaton@adhb.govt.nz

Received

24 November 2005

Accepted 19 May 2006

Published Online First

12 June 2006
Results: Thirty eight patients (63\% men) of mean (SD) age 73.5 (8.04) years and mean (SD) forced expiratory volume in 1 second $0.77(0.35)$ I were evaluated. Overnight desaturation occurred in six $16 \%$; $95 \% \mathrm{Cl} 4$ to 27). Desaturators had mean (SD) resting oxygen saturation on room air of $88(4.2) \%$ compared with $90(4.1) \%$ in non-desaturators $(p=0.15)$, and corrected saturations of $93(2.0) \%$ versus 94 $(2.0) \%(p=0.18)$. HRQL and sleep quality were poor but did not differ between desaturators and nondesaturators.

Conclusions: Most patients did not exhibit overnight desaturation despite not increasing their LTOT prescription overnight. These results challenge the recommendation of routinely increasing overnight oxygen flow in patients receiving LTOT.
$\mathrm{C}$ urrent guidelines recommend increasing the oxygen flow by $1 \mathrm{l} / \mathrm{min}$ during sleep to prevent overnight desaturation in all patients established on long term oxygen therapy (LTOT). The evidence base for this is uncertain, ${ }^{1}$ although hypoxaemia during sleep is well documented $^{23}$ as a consequence of assuming a supine position $^{4}$ and the relative hypoventilation of sleep. ${ }^{5}$ While adjusting oxygen flows at night is easy to advise and may appear easy to perform, in practice this is not necessarily the case. This population is often elderly with considerable comorbidities, and there is the potential for prescription flow rate errors-specifically, inadvertent higher flow rates may cause carbon dioxide retention.

Health-related quality of life (HRQL) ${ }^{67}$ and sleep quality ${ }^{8}$ are poor in patients with chronic obstructive pulmonary disease (COPD) with chronic respiratory failure. Poor sleep quality is probably multifactorial, but overnight desaturation is a possible contributor. LTOT improves HRQL, ${ }^{9}$ but the impact of LTOT on sleep quality remains uncertain.

The aims of this study of COPD patients on LTOT were to determine (1) the prevalence of overnight desaturation if the usual oxygen flow rate is not increased at night, (2) whether resting oxygen saturation predicts overnight desaturation, and (3) whether overnight desaturation correlates with HRQL and sleep quality.

\section{METHODS}

\section{Patient selection}

A cross sectional prospective study was undertaken of consecutive COPD patients established on LTOT attending the outpatient clinic of our regional oxygen outpatient service based in a major metropolitan hospital in Auckland, New Zealand.
The study was classified as an audit of current clinical care by the Auckland Ethics Committee and written informed consent was not required.

\section{Inclusion criteria}

- Primary diagnosis of moderate or severe COPD as defined by British Thoracic Society criteria (forced expiratory volume in 1 second $\left(\mathrm{FEV}_{1}\right)<60 \%$ predicted; ratio of $\mathrm{FEV}_{1}$ to forced vital capacity (FVC) $\left.<70 \%\right) .{ }^{10}$

- Originally fulfilled criteria for LTOT defined as a resting $\mathrm{PaO}_{2}$ either $<7.3 \mathrm{kPa}$ or $\mathrm{PaO}_{2} 7.3-8.0 \mathrm{kPa}$ with evidence of end organ damage at the time of LTOT initiation. End organ damage defined by presence of polycythaemia (PCV $>0.55$ ) or clinical evidence of significant right heart disease either clinically or by ECG ( $p$ wave $>3 \mathrm{~mm}$ in lead II) or evidence of pulmonary hypertension on echocardiogram. LTOT has previously been prescribed at a flow rate titrated to achieve a resting oxygen saturation of $>90 \%$ according to ATS/ERS guidelines. ${ }^{1}$

- Established on LTOT therapy for at least 3 months.

- Clinically stable for at least the previous 4 weeks with no exacerbations. An exacerbation was defined as worsening of respiratory symptoms that required treatment with oral corticosteroids or antibiotics (or both) by the attending physician.

\footnotetext{
Abbreviations: COPD, chronic obstructive pulmonary disease; $\mathrm{FEV}_{1}$, forced expiratory volume in 1 second; FVC, forced vital capacity; HRQL, health-related quality of life; LTOT, long term oxygen therapy; $\mathrm{PaO}_{2}$, arterial oxygen tension
} 


\section{Exclusion criteria}

- Inability to attend hospital outpatient clinic.

- Inability to complete HRQL questionnaires.

- Previous diagnosis of obstructive sleep apnoea and/or the use of nocturnal continuous positive airway pressure.

\section{Data collection}

Data on patient demographic characteristics, significant comorbidity, smoking history, indication for commencing LTOT, and duration of LTOT were collected. Spirometric tests were performed to ATS standards ${ }^{3}$ and $\mathrm{FEV}_{1}$ and FVC were expressed as percentage of predicted normal using the European Community Coal and Steel data set. ${ }^{11}$ Oxygen saturations were measured using finger oximetry (Siemens Micro2 portable oximeter, Siemens Medical Systems; PA, USA) when seated after $\geqslant 30$ minutes at rest, both on room air and their usual oxygen flow. The SF-36 Health Survey Questionnaire (SF-36) ${ }^{12}$ was used to measure generic HRQL, the Chronic Respiratory Questionnaire $(\mathrm{CRQ})^{13}$ was used to measure disease-specific HRQL, and the Pittsburgh Sleep Quality Index (PSQI) ${ }^{14}$ was administered to measure subjective sleep quality and the effects of this on daytime functioning.

Overnight oximetry was performed on two consecutive nights at home. ${ }^{15}$ Overnight desaturation was defined as desaturation below $90 \%$ for $\geqslant 30 \%$ of the night on either of the two nights. ${ }^{16} 17$

\section{Statistical analysis}

The results are presented as mean (SD) values or frequencies and percentages. Continuous variables were compared using an unpaired $t$ test and categorical variables with a $\chi^{2}$ test, except where $25 \%$ of the cells had expected counts of less than 5, in which case the Fisher's exact test was used. p values of $<0.05$ were regarded as being statistically significant.

\section{RESULTS}

Over a 9 month period 61 patients attended the clinic for routine follow up; 23 did not fulfil inclusion criteria (16 clinically unstable, 7 unable to complete questionnaire). The remaining 38 patients were evaluated.

Patient characteristics at the time of assessment in the follow up clinic are shown in table 1 . Their LTOT prescriptions were as follows: $1 \mathrm{l} / \mathrm{min}, \mathrm{n}=20(53 \%) ; 1.5 \mathrm{l} / \mathrm{min}, \mathrm{n}=1 \mathrm{l}$ (39\%); $2 \mathrm{l} / \mathrm{min}, \mathrm{n}=5$ (13\%); $2.5 \mathrm{l} / \mathrm{min}, \mathrm{n}=2$ (5\%). On their usual LTOT flow rate, all patients had resting oxygen saturations $>90 \%$ with 36 patients $(95 \%) \geqslant 92 \%$. Only six patients (16\%; 95\% CI 4 to 27) had overnight desaturation; five ( $13 \%$ ) met the criterion on both nights.

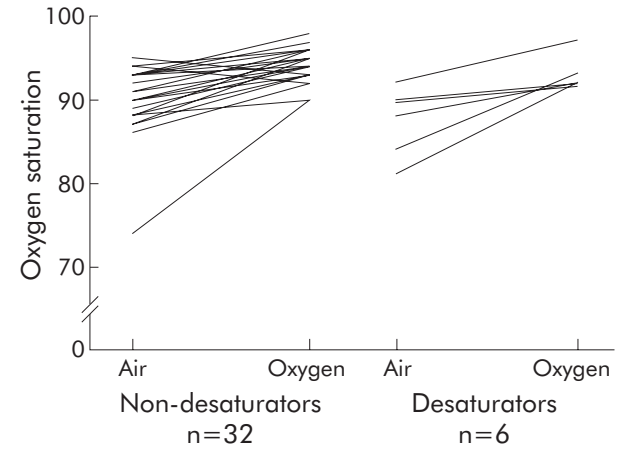

Figure 1 Oxygen saturation on air and oxygen in desaturators and non-desaturators at the time of assessment in the clinic.

Mean (SD) $\mathrm{PaO}_{2}$ at the commencement of LTOT was 6.7 $(0.7) \mathrm{kPa}$ for desaturators and $6.9(0.6) \mathrm{kPa}$ for nondesaturators. Four of the six patients in the desaturator group $(67 \%)$ and 21 of the 32 in the non-desaturator group $(66 \%)$ qualified for LTOT solely on the basis of a $\mathrm{PaO}_{2}$ $<7.3 \mathrm{kPa}$.

At the time of assessment the desaturator group had spent a mean (SD) of $1.2(0.8)$ years on LTOT and the nondesaturator group $2.4(0.8)$ years $(p=0.11)$. Compared with non-desaturators, desaturators had a mean (SD) resting oxygen saturation on room air of $88(4.2) \%$ versus $90(4.1) \%$ $(\mathrm{p}=0.15)$ and corrected mean (SD) oxygen saturation of 93 $(2.0) \%$ versus $94(2.0) \%(p=0.18)$. Only one patient with a corrected oxygen saturation $>94 \%$ had overnight desaturation (fig 1).

HRQL and sleep quality was poor (mean PSQI 7.0 (3.9), mean total CRQ 84.9 (16.9)) but did not differ significantly between desaturators and non-desaturators (table 2).

\section{DISCUSSION}

Current guidelines recommend routinely increasing the oxygen flow rate by $1 \mathrm{l} / \mathrm{min}$ during sleep to prevent overnight desaturation in patients on LTOT. However, the evidence for this recommendation is unclear. ${ }^{1}$ Despite not undertaking this increase in oxygen flow overnight, only a small proportion of our consecutive unselected COPD patients established on LTOT for 3 months or more had overnight desaturation. These results suggest that, for the majority of patients on LTOT, an increase in oxygen flow overnight is not necessary, thus considerably simplifying the regimen and lessening the chance of patient error. The recent ATS/ERS guidelines for dose adjustment of overnight oxygen therapy need to be re-considered in light of these findings.

The reported prevalence of overnight desaturation in COPD patients on LTOT varies widely. In part, this may relate to the

\begin{tabular}{|c|c|c|c|}
\hline & $\begin{array}{l}\text { Desaturators } \\
(n=6)\end{array}$ & $\begin{array}{l}\text { Non-desaturators } \\
(\mathrm{n}=32)\end{array}$ & $\mathrm{p}$ value \\
\hline Male & $6(100 \%)$ & $18(56 \%)$ & 0.07 \\
\hline Age (years) & $71.0(10.18)$ & $73.9(7.68)$ & 0.41 \\
\hline Ethnicity: European & $4(67 \%)$ & $26(81 \%)$ & 0.17 \\
\hline Smoking history (pack years) & $50.5(33.01)$ & $50.8(25.01)$ & 0.98 \\
\hline $\mathrm{FEV}_{1}(\mathrm{I})$ & $0.8(0.20)$ & $0.8(0.37)$ & 0.65 \\
\hline FVC (I) & $2.1(0.65)$ & $1.8(0.52)$ & 0.26 \\
\hline Body mass index $\left(\mathrm{kg} / \mathrm{m}^{2}\right)$ & $26.2(9.70)$ & $25.6(7.95)$ & 0.88 \\
\hline Oxygen saturation on air & $87.5(4.18)$ & $90.2(4.08)$ & 0.15 \\
\hline Oxygen saturation on oxygen & $93.0(2.00)$ & $94.2(1.96)$ & 0.18 \\
\hline Duration on LTOT (years) & $1.2(0.8)$ & $2.3(1.7)$ & 0.11 \\
\hline
\end{tabular}




\begin{tabular}{|c|c|c|c|c|}
\hline & $\begin{array}{l}\text { Non-desaturators } \\
(n=32)\end{array}$ & $\begin{array}{l}\text { Desaturators } \\
(n=6)\end{array}$ & $\begin{array}{l}\text { Mean difference } \\
(95 \% \mathrm{Cl})\end{array}$ & $p$ value \\
\hline \multicolumn{5}{|l|}{ SF-36 } \\
\hline Physical functioning & $26.1(20.94)$ & $17.5(17.54)$ & $8.6(-9.9$ to 27.1$)$ & 0.35 \\
\hline Role limitations - physical & $35.9(37.53)$ & $16.7(12.91)$ & $19.3(-12.5$ to 51.0$)$ & 0.23 \\
\hline Bodily pain & $76.4(22.52)$ & $73.2(25.16)$ & $3.2(-17.5$ to 23.9$)$ & 0.75 \\
\hline General medical health & $37.4(21.01)$ & $41.0(18.63)$ & $-3.6(-22.3$ to 15.0$)$ & 0.70 \\
\hline Vitality & $43.4(17.20)$ & 50.8 (13.93) & $-7.4(-22.5$ to 7.7$)$ & 0.33 \\
\hline Social functioning & $62.9(28.14)$ & $75.0(31.62)$ & $-12.1(-37.9$ to 13.8$)$ & 0.35 \\
\hline Role limitation - emotional & $67.7(41.03)$ & $88.9(17.21)$ & $-21.2(-56.0$ to 13.7$)$ & 0.23 \\
\hline Mental health & 72.1 (17.93) & $76.0(15.18)$ & $-3.9(-19.7$ to 12.0$)$ & 0.62 \\
\hline CRQ Total* $(20-140)$ & $83.7(17.36)$ & 91.5 (13.23) & $-7.8(-23.0$ to 7.4$)$ & 0.30 \\
\hline Dyspnoea (5-25) & $17.5(5.16)$ & $20.2(4.12)$ & $-2.6(-7.2$ to 1.9$)$ & 0.25 \\
\hline Emotional $(7-49)$ & $32.9(7.74)$ & $36.2(8.13)$ & $-3.3(-10.3$ to 3.7$)$ & 0.35 \\
\hline Fatigue $(4-28)$ & $14.2(4.24)$ & $16.0(8.13)$ & $-1.8(-5.4$ to 1.8$)$ & 0.32 \\
\hline Mastery (4-28) & $19.0(5.42)$ & $16.0(1.67)$ & $-0.1(-4.7$ to 4.4$)$ & 0.95 \\
\hline PSQI Total † & $7.1(3.99)$ & $6.7(3.78)$ & $0.4(-3.2$ to 4.0$)$ & 0.82 \\
\hline
\end{tabular}

*Higher scores in CRQ and SF-36 indicate better HRQL. tPSQ Total $>5$ indicative of poor sleep.

variety of definitions of overnight desaturation in use. ${ }^{16} 1819$ In this study we used the widely accepted definition of the Royal College of Physicians Domiciliary Oxygen Therapy Services guidelines. ${ }^{17}$ Overnight desaturation was defined as oxygen saturation below $90 \%$ for $\geqslant 30 \%$ of the nights. One strength of our study was the performance of overnight oximetry on two consecutive nights. However, although we have previously demonstrated considerable between-night variability in overnight desaturation in COPD patients (particularly when mean oxygen saturations were close to $90 \%),{ }^{15}$ in this study the majority of patients $(5 / 6 ; 83 \%)$ who met the criterion for desaturation did so on both nights.

All patients in this study were initially prescribed oxygen at a flow to maintain oxygen saturations at rest $>90 \%$. We would suggest that careful attention to initial dose setting and ensuring that patients are clinically stable may preclude the need for routine up-titration at night. ${ }^{20}$ At the time of this study the mean (SD) time on LTOT of our cohort was 2.2 (1.6) years and a significant proportion (56\%) had resting saturation $\geqslant 94 \%$ on oxygen. The extent to which this may represent the "reparative effects" of long term oxygen as described by $\mathrm{O}^{\prime}$ Donohue ${ }^{21}$ is not clear, and the pathophysiological basis for this remains uncertain. It is possible that the conclusions derived from this study may only be applicable to subjects who have been on LTOT for prolonged periods. However, it needs to be borne in mind that the patients in this study had never increased oxygen flow at night and thus any "reparative effects" of oxygen occurred without the recommended nocturnal increase in flow. Furthermore, 82\% were using a flow rate of less than $2 \mathrm{l} / \mathrm{min}$.

The current recommendation of routinely increasing the oxygen flow rate by $1 \mathrm{l} / \mathrm{min}$ during sleep has considerable implications. While adjusting oxygen flows at night is easy to advise and may appear easy to perform, in practice this is not necessarily the case. This population is often elderly with considerable co-morbidities which may include cognitive, visual, and musculoskeletal impairment, and increasing nocturnal oxygen flow rate may be associated with considerable anxiety. There is also the potential for errors in prescribed nocturnal flow-specifically, inadvertent higher flow rates leading to carbon dioxide retention. We believe that the impact on patients and their caregivers of performing daily flow adjustments may be significantly underestimated. It appears that adherence with this recommendation may be highly variable. ${ }^{22}$ Given that our study showed that the majority of LTOT patients do not require up-titration overnight if careful attention is paid to correcting daytime resting oxygen saturation to $>90 \%$, we would argue that overnight oximetry to ensure adequate correction is eminently easier than indefinite commitment to daily flow adjustments in this patient group.

The low prevalence of overnight desaturation in the present study contrasts with the results of Plywaezewski et al who performed overnight oximetry on 82 patients with COPD eligible for LTOT. ${ }^{23}$ Although oxygen flow was titrated to obtain a resting oxygen saturation $>90 \%, 47.6 \%$ of patients spent $>30 \%$ of the night with an oxygen saturation $<90 \%$. The authors concluded that this supported the need for increased oxygen flow during sleep in about half of COPD patients on LTOT. However, their study is not directly comparable in that the clinical stability of their patients was not specifically stated and they had not been established on LTOT. The mean (SD) overnight oxygen saturation on oxygen in this study was 90.9 (5.0)\% compared with 93.3 $(3.0) \%$ in our cohort. The low rate of desaturation in our cohort may be due to careful setting of the initial flow rate according to ATS/ERS guidelines, clinical exclusion of patients with obstructive sleep apnoea syndrome, time period on LTOT, as well as the clinical stability of our patients at the time of establishment on LTOT.

Quality of life is increasingly acknowledged as a very important clinical outcome measure for patients with COPD. HRQL and sleep quality was generally poor but did not differ between desaturators and non-desaturators. While acknowledging the limited numbers of desaturators in this study, these data do not suggest detrimental effects of overnight desaturation on HRQL and sleep quality in this patient group. The assessment of sleep quality in patients with COPD is problematic as it may be influenced by a number of other factors including age and co-morbidities. Although the PSQI has been widely used and validated in a number of diseases, ${ }^{14}$ it has not been validated specifically for use in COPD. Appropriate assessment of sleep quality in COPD is not optimal and needs greater attention in future studies.

Our study reports on unselected consecutive patients attending for routine follow up at an oxygen service clinic. While the results are probably generalisable to COPD patients on LTOT who are able to attend clinic, they may not necessarily be applicable to those patients on LTOT who are housebound or those who have recently commenced LTOT. In this study obstructive sleep apnoea syndrome was excluded on the basis of history; routine polysomnography was not undertaken. While this represents a pragmatic, clinically appropriate approach, some patients with obstructive sleep apnoea may be missed and be responsible in part for desaturation observed in those with adequate resting oxygen 
saturations. We acknowledge the small size of our cohort and that our study may be underpowered to detect significant differences between desaturators and non-desaturators, and therefore the results need to be interpreted with care.

In conclusion, most patients with COPD on LTOT did not exhibit overnight desaturation despite not increasing their usual LTOT prescription overnight. These results challenge the current recommendations of routinely increasing oxygen flow overnight in all patients established on LTOT. Larger prospective studies are required to clarify whether there is a need for routine up-titration of overnight flow in COPD patients on LTOT in whom the initial flow corrected resting daytime saturations, and whether such changes lead to improved patient outcomes.

\section{ACKNOWLEDGEMENTS}

The authors acknowledge the contribution of Jenny Hicks, Jean Merrington, Pam Sherlock (oxygen nurse specialists) and Teena West (biostatistician).

\section{Authors' affiliations}

M Nisbet, T Eaton, C Lewis, W Fergusson, J Kolbe, Green Lane Respiratory Services, Auckland City Hospital, University of Auckland, Auckland, New Zealand

J Kolbe, Department of Medicine, Faculty of Health Sciences, University of Auckland, Auckland, New Zealand

Funding: none.

Competing interests: none.

\section{REFERENCES}

1 Celli B, MacNee W. Standards for the diagnosis and care of patients with COPD: a summary of the ATS/ERS position paper. Eur Respir J 2004;23:932-46.

2 Douglas N, Calverley P, Leggett R, et al. Transient hypoxaemia during sleep in chronic bronchitis and emphysema. Lancet 1979;1:1-4.

3 Stradling J, Lane D, Nocturnal hypoxaemia in chronic obstructive pulmonary disease. Clin Sci 1983;64:213-22.

4 Hudgel D, Martin R, Johnson B, et al. Mechanics of the respiratory system and breathing pattern during sleep in normal humans. J Appl Physiol

1984;56:133-7.
5 Catterall J, Calverley P, MacNee W, et al. Mechanism of transient nocturnal hypoxaemia in hypoxic chronic bronchitis and emphysema. J Appl Physiol 1985;59:1698-703.

6 Curtis J, Deyo R, Hudson L. Health-related quality of life among patients with chronic obstructive pulmonary disease. Thorax 1994:49:162-70.

7 Okubadejo A, Jones P, Wedzicha J. Quality of life in patients with chronic obstructive pulmonary disease and severe hypoxaemia. Thorax 1996;51:44-7

8 Cormick W, Olson L, Hensley M, et al. Nocturnal hypoxaemia and quality of sleep in patients with chronic obstructive lung disease. Thorax 1986;41:846-54.

9 Eaton T, Lewis C, Young P, et al. Long-term oxygen therapy improves healthrelated quality of life. Respir Med 2004;98:285-93.

10 COPD Guidelines Group of the Standards of Care Commitee of the BTS. BTS guidelines for the management of chronic obstructive pulmonary disease. Thorax 1997;52(Suppl 5):S13-4.

11 Quanier P. Standardisation of lung function testing. Report of working party European Community for Coal and Steel recommendations. Bull Eur Physiopathol Respir 1983;19(Suppl 5):1-93.

12 Ware J, Snow K, Kosinski M, et al. SF-36 health survey: manual and interpretation guide. Boston: The Health Institute, 1993.

13 Guyatt G, Berman L, Townsend $M$, et al. A measure of quality of life for clinical trials in chronic lung disease. Thorax 1987:42:773-8.

14 Buysse D, Reynolds C, Monk T, et al. The Pittsburgh sleep quality index: a new instrument for psychiatric practice and research. Psychiatry Res 1989;28:193-213.

15 Lewis C, Eaton T, Fergusson W, et al. Home overnight pulse oximetry in patients with COPD - more than one recording may be needed. Chest 2003;123:1127-33.

16 Chaouat A, Weitzenblum E, Kessler R, et al. Sleep-related oxygen desaturation and daytime pulmonary haemodynamics in COPD patients with mild hypoxaemia. Eur Respir J 1997;10:1730-5.

17 Wedzicha J. Domiciliary oxygen therapy services: clinical guidelines and advice for prescribers. Summary of a report of the Royal College of Physicians. J R Coll Physicians Lond 1999;33:445-7.

18 Fletcher E, Miller J, Divine G, et al. Nocturnal oxyhaemoglobin desaturation in COPD patients with arterial oxygen tensions above $60 \mathrm{mmHg}$. Chest 1992:4:604-8.

19 Vos $\mathrm{P}$, Folgering $\mathrm{H}$, van Herwaarden $\mathrm{C}$. Predictors for nocturnal hypoxaemia (mean $\mathrm{SaO}_{2}<90 \%$ ) in normoxic and mildly hypoxic patients with COPD. Eur Respir J 1995;8:74-7.

20 Guyatt G, Nonoyama M, Lacchetti C, et al. A randomised trial of strategies for assessing eligibility for long-term domiciliary oxygen therapy. Am J Respir Crit Care Med 2005; 172:573-80

21 O'Donohue W. Effect of oxygen therapy on increasing arterial oxygen tension in hypoxemic patients with stable chronic obstructive pulmonary disease while breathing ambient air. Chest 1991;100:968-72.

22 Wijkstra P, Guyatt G, Ambosino N, et al. International approaches to the prescription of long-term oxygen therapy. Eur Respir J 2001;18:909-13.

23 Plywaezewski R, Sliwinski P, Nowinski A, et al. Incidence of nocturnal desaturation while breathing oxygen in COPD patients undergoing long-term oxygen therapy. Chest 2000;117:679-83. 